\title{
Clinical characteristics associated with occurrence and poor prognosis of interstitial lung disease in rheumatoid arthritis
}

Ji Ae Yang ${ }^{*}$ Jeong Seok Lee*, Jin Kyun Park, Eun Bong Lee, Yeong Wook Song, and Eun Young Lee

Division of Rheumatology, Department of Internal Medicine, Seoul National University College of Medicine, Seoul, Korea

\author{
Received: February 15, 2016 \\ Revised : April 11, 2016 \\ Accepted: May 3, 2016

\section{Correspondence to} \\ Eun Young Lee, M.D. \\ Division of Rheumatology, \\ Department of Internal \\ Medicine, Seoul National \\ University College of Medicine, \\ 101 Daehak-ro, Jongno-gu, Seoul \\ 03080, Korea \\ Tel: +82-2-2072-0852 \\ Fax: $+82-2-762-9662$ \\ E-mail: elee@snu.ac.kr
}

\section{${ }^{*}$ These authors contributed} equally to this work.
Background/Aims: To analyze clinical characteristics of interstitial lung disease (ILD) associated with rheumatoid arthritis (RA), especially in patients with poor prognosis.

Methods: Seventy-seven RA patients with ILD and 231 age, sex, and disease duration-matched RA patients without ILD were enrolled in this retrospective study. Epidemiologic, clinical, and laboratory information were obtained through a medical chart review. Logistic regression analysis was used to estimate the risk of mortality in RA patients with ILD.

Results: Compared to the RA without ILD group, the RA with ILD group had significantly higher titers of rheumatoid factor and the anti-cyclic citrullinated peptide ( $p=0.001$ for both), higher levels of C-reactive protein (CRP) at the time of RA diagnosis $(p=0.014)$, and a higher erythrocyte sedimentation rate $(p=0.022)$ and CRP levels $(p<0.001)$ throughout the 10-year follow-up period. These patients also received a higher mean daily dose of corticosteroids $(p<0.001)$. In the subgroup analysis of RA patients with ILD, 28 patients (36.4\%) died during follow-up. Multivariate analysis revealed that older age at the time of ILD diagnosis was significantly associated with mortality. Usual interstitial pneumonia (UIP) subtype on high-resolution computed tomography (HRCT) was also suggested as a poor prognostic factor.

Conclusions: The survival of RA patients with ILD is adversely affected by age at the time of ILD diagnosis. RA-ILD patients diagnosed after age 65 or with a UIP subtype on HRCT may have a poor prognosis.

Keywords: Arthritis, rheumatoid; Lung diseases, interstitial; Rheumatoid arthritis-associated interstitial lung disease

\section{INTRODUCTION}

Rheumatoid arthritis (RA)-associated interstitial lung disease (ILD) contributes significantly to the morbidity and mortality of affected patients [1]. However, the presence of ILD has been largely ignored in the management of RA, because the symptoms of ILD are subclini- cal in most patients [2].

Recent studies have indicated that ILD may be a feature of early RA and its prevalence increases with age [3]. Usual interstitial pneumonia (UIP) is the most common subtype of ILD in RA patients detected on high-resolution computed tomography (HRCT), and importantly, this subtype of ILD is associated with a higher risk of 
mortality in RA compared to patients with other forms of ILD related to connective tissue disease. In fact, the survival rate of RA patients with UIP is similar to that of patients with idiopathic pulmonary fibrosis, which is the most devastating form of ILD $[1,4,5]$. Given the complexities of these two diagnoses, a multidisciplinary approach is recommended for the management of ILD in RA patients. Most knowledge regarding the progression and prognosis of patients with ILD related to connective tissue disease is derived from studies of the natural history of patients with systemic sclerosis-associated ILD. It remains to be investigated whether these data are applicable to other forms of ILD related to connective tissue disease [6]. The management of RA patients is complicated by the use of several drugs with potential lung toxicity in the presence of ILD. Although there is limited evidence at present, these agents should be used with caution in RA patients with $\operatorname{ILD}[7,8]$.

In this study, we identified clinical characteristics associated with ILD occurrence and its poor prognosis.

\section{METHODS}

\section{Patients}

Seventy-seven patients with RA and ILD who received longitudinal clinical care at Seoul National University Hospital from January 1991 to December 2011 were enrolled. The diagnosis of RA was based on the 1987 revised classification criteria of the American College of Rheumatology [9]. ILD was diagnosed according to American Thoracic Society criteria, which included consistent clinical features and pulmonary function tests, radiographic evidence of interstitial disease, and/ or lung histopathology that was consistent with this diagnosis [10]. As a reference group, 231 age- or sex- and disease duration-matched RA patients without ILD who received medical care during the same period were randomly selected from the medical record archive at a ratio of 1 to 3. The study was approved by the Institutional Review Board of Seoul National University Hospital (approval no. 1310-089-528) and was conducted in accordance with the principles of the Declaration of Helsinki and Good Clinical Practice guidelines. The requirement for patient consent was waived by the IRBs due to the retrospective character of the study.

\section{Outcome measurements}

Medical records of the selected patients were reviewed. The epidemiologic, clinical, and laboratory data were examined. Rheumatoid factor (RF) was measured by immunoturbidimetric assay (reference range, < $15 \mathrm{IU} / \mathrm{mL}$ ), and anti-cyclic citrullinated peptide (anti-CCP) was measured by chemiluminescent microparticle immunoassay (reference range, $<5.0 \mathrm{IU} / \mathrm{mL}$ ). Data on the levels of erythrocyte sedimentation rate (ESR; reference range, < $20 \mathrm{~mm} / \mathrm{hr}$ ) and C-reactive protein (CRP; reference range, $<0.5 \mathrm{mg} / \mathrm{dL}$ ) were collected annually since the time of RA diagnosis. The presence of erosion on joint radiographs and the mean daily corticosteroid dose were examined as measures of RA outcome over the 10-year follow-up period after RA diagnosis in the two groups. In order to identify the risk factors for mortality in the RA with ILD group, survival data were obtained from the Ministry of Security and Public Administration.

\section{Statistical analysis}

The Student $t$ test for continuous variables and chisquare or Fisher exact test for categorical variables were used to compare the patients with and without ILD. The results were reported as mean \pm standard deviation. Binary logistic regression analysis was used to identify the risk factors for mortality in the RA-ILD group. The results were expressed as adjusted odds ratios (ORs) with 95\% confidence intervals (CIs). Survival was analyzed with the Kaplan-Meier method and compared with the log-rank test. Two-sided $p$ values less than 0.05 were considered statistically significant. All of the analyses were performed with IBM SPSS version 21 (IBM Corp., Armonk, NY, USA).

\section{RESULTS}

\section{Comparison of the clinical characteristics between the RA patients with and without ILD}

The study cohort comprised 77 RA patients with ILD and 231 age-, sex-, and disease duration-matched RA patients without ILD. The proportions of ex-smokers, current smokers, and their pack-years did not differ significantly between groups. A history of tuberculosis or nontuberculous mycobacteria (NTM) was found more frequently in RA patients with ILD (Table 1). 
RF and anti-CCP positivity did not differ significantly between groups. However, RA patients with ILD had higher RF (521.1 \pm 828.3 vs. $249.3 \pm 332.4, p=0.001)$ and anti-CCP titers $(242.8 \pm 234.4$ vs. $125.3 \pm 144.3, p=0.001)$ than those of RA control group. The baseline ESR did not differ significantly between the RA patients with and without ILD $(59.9 \pm 37.3 \mathrm{~mm} / \mathrm{hr}$ vs. $44.9 \pm 35.1 \mathrm{~mm} / \mathrm{hr}$, respectively). However, the baseline CRP levels in the RA with ILD group $(29.5 \pm 35.3 \mathrm{mg} / \mathrm{L})$ were significantly higher than those in RA without ILD group (19.1 \pm 29.5 , $p=0.014)$. The percentages of RA patients with erosive change at baseline did not differ significantly between the two groups (Table 1). The medications-including disease-modifying antirheumatic drugs (DMARDs) and biologic agents-differed significantly between the two groups. This difference might be due to the concerns of lung toxicity provoked by certain drugs. More than two-thirds of RA patients without ILD received methotrexate (MTX; $\mathrm{n}=176,76.2 \%$ ), compared with only one-third ( $\mathrm{n}=30,39.0 \%, p<0.001)$ of RA patients with ILD. The proportions of patients receiving sulfasalazine (63.2\% vs. $32.0 \%, p<0.001)$ and rituximab (2.6\% vs. $\% \%$,

Table 1. Clinical characteristics of RA patients with and without ILD

\begin{tabular}{|c|c|c|c|}
\hline Characteristic & RA with ILD (n = 77) & RA without ILD (n = 231) & $p$ value \\
\hline Age, yr & $56.6 \pm 13.1$ & $57.1 \pm 11.7$ & $0.731^{\mathrm{a}}$ \\
\hline Female sex & $58(75 \cdot 3)$ & $174(75 \cdot 3)$ & $1.000^{\mathrm{b}}$ \\
\hline Disease duration, yr & $11.5 \pm 8.5$ & $10.8 \pm 6.9$ & $0.45^{\mathrm{a}}$ \\
\hline \multicolumn{4}{|l|}{ Smoking } \\
\hline Ex-smoker & $10 / 68(14.7)$ & $18 / 152(11.8)$ & $0.162^{b}$ \\
\hline Current smoker & $4 / 68(5 \cdot 9)$ & $7 / 152(4.6)$ & $0.367^{c}$ \\
\hline Pack-years & $28.4 \pm 19.8$ & $33.7 \pm 15 \cdot 4$ & $0.445^{\mathrm{a}}$ \\
\hline History of tuberculosis or NTM & $12(15.6)$ & $16(6.9)$ & $0.022^{b}$ \\
\hline \multicolumn{4}{|l|}{ Serology } \\
\hline RF positivity & $56 / 76(73 \cdot 7)$ & $160 / 227(70.5)$ & $0.838^{b}$ \\
\hline RF titer & $521.1 \pm 828.3$ & $249 \cdot 3 \pm 332.4$ & $0.001^{\mathrm{a}}$ \\
\hline Anti-CCP positivity & $33 / 43(76.7)$ & $95 / 142(66.9)$ & $0.335^{b}$ \\
\hline Anti-CCP titer & $242.8 \pm 234 \cdot 4$ & $125 \cdot 3 \pm 144 \cdot 3$ & $0.001^{\mathrm{a}}$ \\
\hline Baseline ESR, mm/hr & $59 \cdot 9 \pm 37 \cdot 3$ & $44 \cdot 9 \pm 35.1$ & $0.193^{\mathrm{a}}$ \\
\hline Baseline CRP, mg/L & $29 \cdot 5 \pm 35 \cdot 3$ & $19.1 \pm 29.5$ & $0.014^{\mathrm{a}}$ \\
\hline Presence of erosion on joint X-rays at baseline & $77(33 \cdot 3)$ & $15(19 \cdot 5)$ & $0.125^{\mathrm{b}}$ \\
\hline Medication & 77 & 231 & \\
\hline Methotrexate & $30(39.0)$ & $176(76.2)$ & $<0.001^{\mathrm{b}}$ \\
\hline Leflunomide & $22(28.6)$ & $60(26.1)$ & $0.670^{b}$ \\
\hline Hydroxychloroquine & $46(59 \cdot 7)$ & $147(63.6)$ & $0.540^{b}$ \\
\hline Sulfasalazine & $48(63.2)$ & $73(32.0)$ & $<0.001^{b}$ \\
\hline Tacrolimus & $2(2.6)$ & $6(2.6)$ & $0.991^{c}$ \\
\hline TNF inhibitor & $4(5.2)$ & $12(5.2)$ & $1.000^{c}$ \\
\hline Rituximab & $2(2.6)$ & 0 & $<0.001^{\mathrm{c}}$ \\
\hline
\end{tabular}

Values are presented as mean \pm SD or number (\%).

RA, rheumatoid arthritis; ILD, interstitial lung disease; NTM, nontuberculous mycobacteria; RF, rheumatoid factor; anti-CCP, anti-cyclic citrullinated peptide; ESR, erythrocyte sedimentation rate; CRP, C-reactive protein; TNF, tumor necrosis factor.

${ }^{\mathrm{a}}$ Student $t$ test.

${ }^{\mathrm{b}}$ Chi-square test.

${ }^{\mathrm{c}}$ Fisher exact test. 

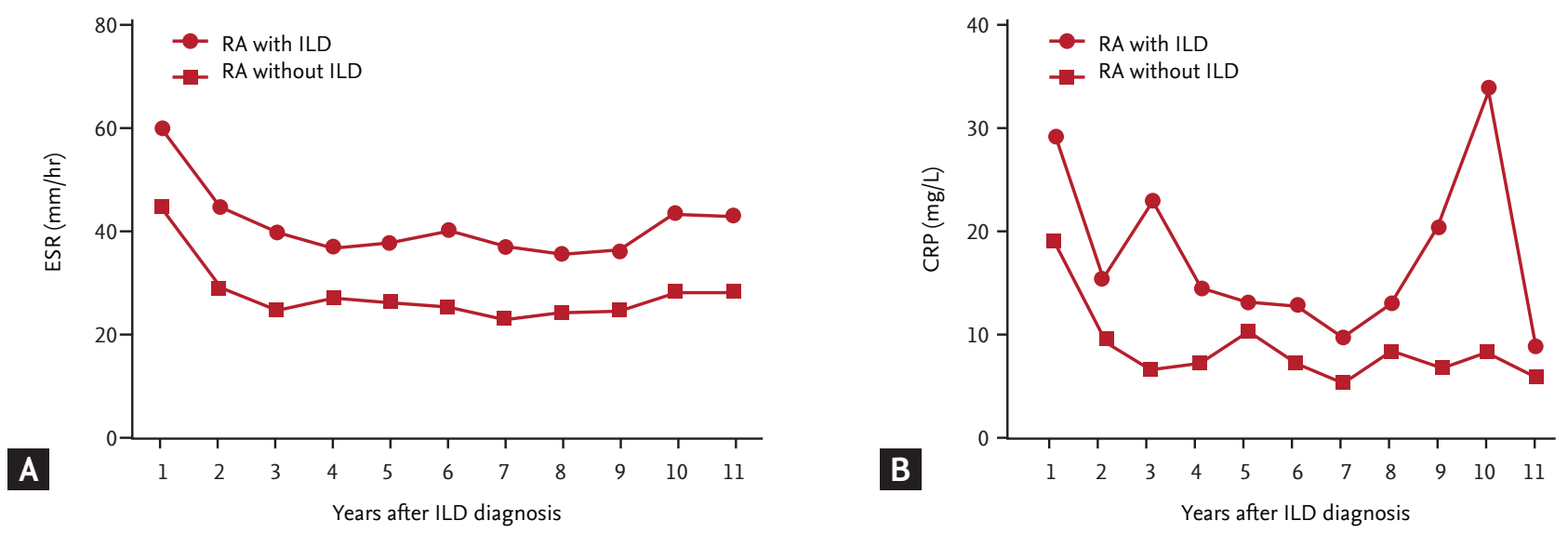

Figure 1. Serial changes in (A) erythrocyte sedimentation rate (ESR) and (B) C-reactive protein (CRP) levels in rheumatoid arthritis (RA) patients with interstitial lung disease (ILD) compared to those without ILD over the 10-year follow-up.

$p<0.001$ ) were significantly higher in the RA with ILD group compared to the RA without ILD group. Use ofleflunomide, hydroxychloroquine, tacrolimus, and tumor necrosis factor inhibitors were similar in the two groups (Table 1).

The mean ESR $(47.9 \pm 25.5 \mathrm{~mm} / \mathrm{hr}$ vs. $31.7 \pm 21.9 \mathrm{~mm} / \mathrm{hr}$, $p=0.022)$ and CRP levels $(21.2 \pm 23.8 \mathrm{mg} / \mathrm{L}$ vs. $11.2 \pm 15.4$ $\mathrm{mg} / \mathrm{L}, p<0.001)$ in the RA with ILD group over the 10year follow-up period were significantly higher than those in the RA control group (Fig. 1). This finding suggests that RA patients with ILD had higher inflammatory burdens over the disease course. The RA with ILD group received a higher mean daily dose of corticosteroid than the RA control group $(6.7 \pm 7.6 \mathrm{mg} /$ day vs. $3.6 \pm 2.4 \mathrm{mg} /$ day, respectively; $p<0.001)$.

\section{Subgroup analysis of the RA with ILD group}

\section{Demographic characteristics}

Seventy-seven patients with RA with ILD ( 58 females, 75.3\%) were analyzed. The age at the time of ILD diagnosis was $59.0 \pm 13.3$ years, and patients were followed up for an average of $8.7 \pm 4.9$ years from ILD diagnosis. The number of patients who had articular disease that predated or was synchronous with ILD was 57 (74.0\%). RA patients with ILD had a normal forced vital capacity $(2.34 \pm 0.72 \mathrm{~L}, 81.2 \% \pm 19.7 \%)$ and a mildly decreased diffusing capacity of carbon monoxide (DLCO; 14.06 $\pm 25.95,68.0 \% \pm 20.3 \%)$ at baseline. HRCT showed that 32 patients $(47.8 \%)$ had the UIP subtype, 17 (25.4\%) had the nonspecific interstitial pneumonia (NSIP) subtype, and 13 (19.4\%) had the organizing pneumonia subtype. Sixteen patients underwent lung biopsies, and the distribution of disease was consistent with the diagnosis suggested by HRCT (Table 2).

Risk factors for mortality in RA with ILD in uni- and multivariate analysis

Of the 77 RA patients with ILD, 28 patients (36.4\%) died. We divided RA patients with ILD into two groups according to survival in order to further evaluate the risk factors for mortality. Females had significantly better survival than males $(p=0.025)$, and a high titer of RF was associated with poor survival in the RA with ILD group ( $p=0.001)$. Patients with the UIP subtype on HRCT were significantly associated with death (OR, 5.846; 95\% CI, 1.970 to 17.353 ), whereas patients with the NSIP subtype were associated with lower risk of death than other subtypes (OR, 0.0625; 95\% CI, 0.0077 to 0.508). Survival was not related to baseline lung function, histopathology on the lung biopsies, or medication (Table 2).

Multivariate logistic regression analysis was performed to evaluate the risk factors for mortality while adjusting for confounding variables (Fig. 2). The factor that was independently associated with fatal outcome was older age at the time of ILD diagnosis (OR, 1.08; 95\% CI, 1.02 to 1.15; $p=0.012$ ). In the Kaplan-Meier survival curves that were stratified by the category of HRCT subtype (Fig. 3A), patients with UIP on HRCT had poorer survival than those without UIP ( $p=0.05$, log-rank test). Similarly, as 
Table 2. Demographic characteristics and risk factors for mortality in the RA patients with ILD in univariate analysis

\begin{tabular}{|c|c|c|c|c|}
\hline Characteristic & RA with ILD ( $\mathrm{n}=77)$ & Death $(n=28)$ & Alive $(n=49)$ & $p$ value \\
\hline Age at the time of ILD diagnosis & $59.0 \pm 13.3$ & $64.1 \pm 10.4$ & $56.3 \pm 11.7$ & $0.314^{\mathrm{a}}$ \\
\hline Female sex & $58(75 \cdot 3)$ & $17(60.7)$ & $41(83.7)$ & $0.025^{\mathrm{b}}$ \\
\hline \multicolumn{5}{|l|}{ Smoking } \\
\hline Ex-smoker & $10 / 68(14.7)$ & $6 / 27(22.2)$ & $4 / 41(9.7)$ & $0.096^{\mathrm{c}}$ \\
\hline Current smoker & $4 / 68(5.9)$ & $2 / 27(7.4)$ & $2 / 41(4.9)$ & $0.560^{c}$ \\
\hline Pack-years & $28.4 \pm 19.8$ & $21.4 \pm 12.1$ & $42.5 \pm 26.3$ & $0.154^{\mathrm{a}}$ \\
\hline \multicolumn{5}{|l|}{ Serology } \\
\hline RF positivity & $5 / 76(73.7)$ & $23 / 28(82.1)$ & $34 / 48(70.8)$ & $0.161^{b}$ \\
\hline RF titer & $521.1 \pm 828.3$ & $774.5 \pm 1,112.9$ & $345.2 \pm 506.4$ & $0.001^{\mathrm{a}}$ \\
\hline Anti-CCP positivity & $33 / 43(76.7)$ & $9 / 13(69.2)$ & $24 / 30(80.0)$ & $0.151^{\mathrm{b}}$ \\
\hline Anti-CCP titer & $242.8 \pm 234.4$ & $189.4 \pm 224.5$ & $263.4 \pm 239.1$ & $0.368^{\mathrm{a}}$ \\
\hline Mean ESR, mm/hr & $47 \cdot 9 \pm 25 \cdot 5$ & $58.0 \pm 25.0$ & $42.2 \pm 24.3$ & $0.008^{a}$ \\
\hline Mean CRP, mg/L & $31.7 \pm 21.9$ & $37.2 \pm 37.8$ & $25.1 \pm 33.5$ & $0.009^{\mathrm{a}}$ \\
\hline \multicolumn{5}{|l|}{ Medication } \\
\hline Methotrexate & $30(39.0)$ & $8(28.6)$ & $22(45.8)$ & $0.138^{b}$ \\
\hline Leflunomide & $22(28.6)$ & $7(25.0)$ & $15(31.3)$ & $0.562^{b}$ \\
\hline Hydroxychloroquine & $46(59.7)$ & $17(60.7)$ & $29(60.4)$ & $0.980^{b}$ \\
\hline Sulfasalazine & $48(63.2)$ & $16(57.1)$ & $32(66.7)$ & $0.406^{b}$ \\
\hline Tacrolimus & $2(2.6)$ & $1(3.6)$ & $1(2.1)$ & $0.696^{\mathrm{c}}$ \\
\hline TNF inhibitor & $4(5.2)$ & $1(3.6)$ & $3(6.3)$ & $0.614^{c}$ \\
\hline Rituximab & $2(2.6)$ & $1(3.6)$ & $1(2.1)$ & $0.696^{\mathrm{c}}$ \\
\hline Mean corticosteroid dose, mg/day & $6.7 \pm 7.6$ & $7.1 \pm 8.3$ & $6.4 \pm 7 \cdot 3$ & $0.730^{\mathrm{a}}$ \\
\hline \multicolumn{5}{|l|}{ Baseline lung function } \\
\hline FVC, L & $2.34 \pm 0.72$ & $2.32 \pm 0.78$ & $2.35 \pm 0.69$ & $0.304^{c}$ \\
\hline Predicted, \% & $81.2 \pm 19.7$ & $80.0 \pm 19.9$ & $81.9 \pm 19.9$ & $0.739^{c}$ \\
\hline DLCO & $14.06 \pm 25.95$ & $10.15 \pm 3.13$ & $16.23 \pm 32.20$ & $0.249^{c}$ \\
\hline Predicted, \% & $68.0 \pm 20.3$ & $64 \cdot 5 \pm 21.6$ & $70.1 \pm 19.5$ & $0.600^{c}$ \\
\hline \multicolumn{5}{|l|}{ Subtypes on HRCT } \\
\hline NSIP & $17 / 67(25 \cdot 4)$ & $1 / 26(3.8)$ & $16 / 41(39.0)$ & $0.001^{\mathrm{c}, \mathrm{d}}$ \\
\hline $\mathrm{OP}$ & $13 / 67(19.4)$ & $5 / 26(19.2)$ & $8 / 41(19.5)$ & $0.489^{\mathrm{b}}$ \\
\hline UIP & $32 / 67(47.8)$ & $19 / 26(73.1)$ & $13 / 41(31.7)$ & $0.001^{\mathrm{b}, \mathrm{d}}$ \\
\hline $\mathrm{DPB}$ & $2 / 67(3.0)$ & $0 / 26(0.0)$ & $2 / 41(4.9)$ & $0.371^{c}$ \\
\hline LIP & $2 / 67(3.0)$ & $0 / 26(0.0)$ & $2 / 41(4.9)$ & $0.371^{c}$ \\
\hline DIP & $1 / 67(1.5)$ & $1 / 26(3.8)$ & $0 / 41(0.0)$ & $0.388^{c}$ \\
\hline \multicolumn{5}{|l|}{ Histopathology on lung biopsy } \\
\hline NSIP & $4 / 16(25.0)$ & $0 / 4(0.0)$ & $4 / 12(30.0)$ & $0.272^{c}$ \\
\hline $\mathrm{OP}$ & $2 / 16(12.5)$ & $1 / 4(25.0)$ & $1 / 12(8.3)$ & $0.450^{c}$ \\
\hline UIP & $7 / 16(43.8)$ & $2 / 4(50.0)$ & $5 / 12(41.7)$ & $0.608^{c}$ \\
\hline DPB & $1 / 16(6.3)$ & $0 / 4(0.0)$ & $1 / 12(8.3)$ & $0.750^{c}$ \\
\hline LIP & $1 / 16(6.3)$ & $0 / 4(0.0)$ & $1 / 12(8.3)$ & $0.750^{c}$ \\
\hline DIP & $1 / 16(6.3)$ & $1 / 4(25.0)$ & $0 / 12(0.0)$ & $0.250^{c}$ \\
\hline
\end{tabular}

Values are presented as mean \pm SD or number (\%).

RA, rheumatoid arthritis; ILD, interstitial lung disease; RF, rheumatoid factor; anti-CCP, anti-cyclic citrullinated peptide; ESR, erythrocyte sedimentation rate; CRP, C-reactive protein; TNF, tumor necrosis factor; FVC, forced vital capacity; DLCO, diffusing capacity of carbon monoxide; HRCT, high-resolution computed tomography; NSIP, nonspecific interstitial pneumonia; OP, organizing pneumonia; UIP, usual interstitial pneumonia; DPB, diffuse panbronchiolitis; LIP, lymphocytic interstitial pneumonia; DIP, desquamative interstitial pneumonia.

${ }^{\mathrm{a}}$ Student $t$ test.

${ }^{\mathrm{b}}$ Chi-square test.

${ }^{\mathrm{c}}$ Fisher exact test.

${ }^{\mathrm{d}}$ Following Bonferroni's correction for multiple comparisons, $p<0.05 / 6=0.0083$ is considered significant. These represent significant values even after Bonferroini's correction. 
shown in Fig. 3B, patients who were older ( $\geq 65$ years) at the time of ILD diagnosis had poorer survival than those who were younger $(<65$ years) $(p<0.001$, log-rank test). We identified the cause of death in 13 patients as follows: six deaths from pneumonia, three ILD, two lung cancer, one acute myocardial infarction, and one temporal bone cancer. In this small number of patients for whom cause of death was known, we could not conclude that specific cause of death was significantly associated with UIP pattern on HRCT. However, all patients who died from ILD and lung cancer had UIP patterns on HRCT.

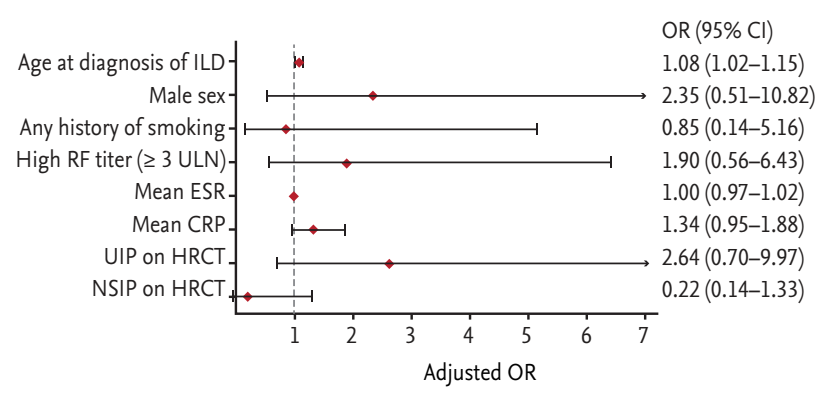

Figure 2. A forest plot showing the association of clinical risk factors and mortality in rheumatoid arthritis-interstitial lung disease (ILD) patients analyzed by binary logistic regression analysis. OR, odds ratio; CI, confidence interval; RF, rheumatoid factor; ULN, upper limit of normal; ESR, erythrocyte sedimentation rate; CRP, C-reactive protein; UIP, usual interstitial pneumonia; HRCT, high-resolution computed tomography; NSIP, nonspecific interstitial pneumonia.

\section{DISCUSSION}

Compared to RA patients without ILD, those with ILD had higher titers of RF and anti-CCP and a more frequent history of tuberculosis or NTM infection. In addition, the RA patients with ILD had higher CRP levels at the time of RA diagnosis and higher mean ESR and CRP levels, which were monitored annually. The latter group had higher inflammatory burdens during the disease course, and they also received a higher mean daily dose of corticosteroids.

RF was reported to be present in high titers in RA patients with ILD [3]. It was also reported that anti-citrullinated peptide antibody (ACPA) positivity correlates with the presence of ILD in RA, and higher titers of ACPA may be associated with more severe ILD $[11,12]$. The current results supported the previous report, but the RF and anti-CCP positivity were similar between RA patients with and without ILD.

The incidence of pulmonary tuberculosis is increased in patients with ILD [13]. In this study, patients with a history of tuberculosis or NTM were found more frequently in the RA with ILD group. It is possible that a patient with a history of pulmonary tuberculosis may have poor lung function and therefore be more likely to receive an incidental diagnosis of ILD during follow-up for their tuberculosis.

Although the ESR and CRP levels correlated with RA disease activity, the roles of these inflammatory markers in the evaluation of lung disease are unclear [14]. A
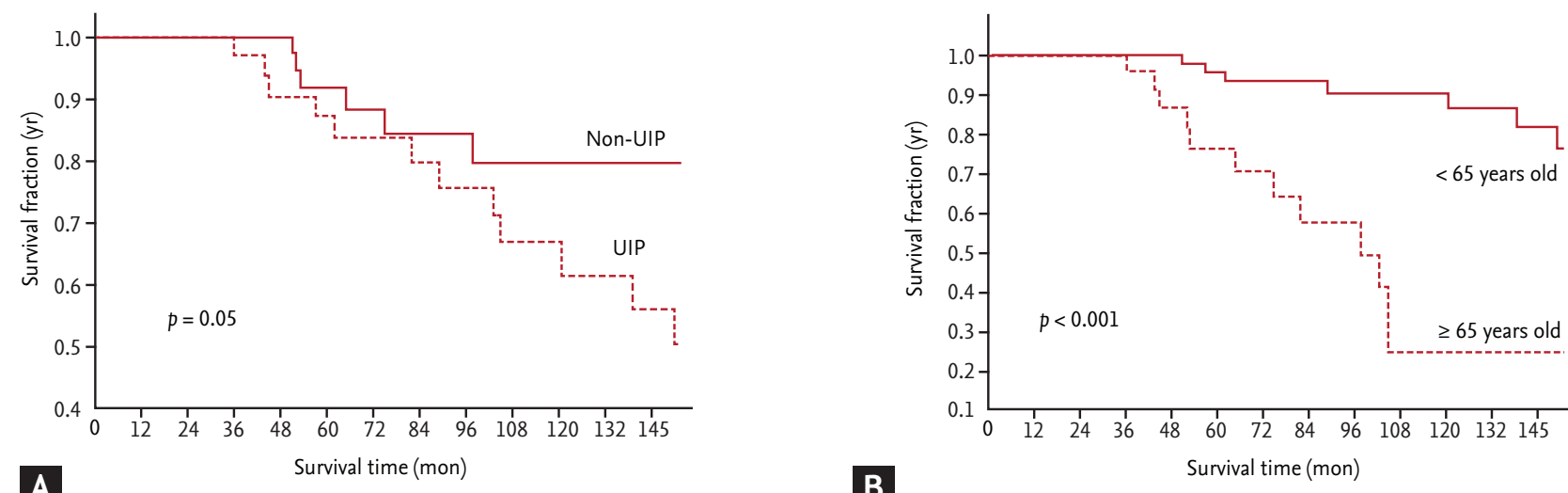

Figure 3. Kaplan-Meier curves of the survival of patients (A) with or without usual interstitial pneumonia (UIP) on high-resolution computed tomography and (B) stratified by age at the time of interstitial lung disease diagnosis $(\geq 65$ years vs. $<65$ years). 
previous study has shown that baseline ESR values were increased in RA patients with ILD [15]. The present study showed that the baseline CRP levels in RA patients with ILD were higher compared with the age- or sex- and disease duration-matched RA patients without ILD. Baseline CRP levels have been suggested to be predictive of long-term ILD progression in patients with systemic sclerosis-associated ILD [16]. CRP is a general marker of inflammation and involved in the innate immune response to systemic inflammation that is downstream from interleukin 6 (IL-6) and IL-1 $\beta$ [17]. IL-6 levels correlated moderately with high-sensitivity CRP levels [18]. Further studies are needed to examine whether IL- 6 or other proinflammatory cytokines have additional predictive significance beyond the CRP levels for development of ILD in RA.

The question of whether there is a causal relationship between poor outcomes in RA and the limited usage of MTX and other DMARDs contributing to lung toxicity is controversial. A recent study reported that disease activity scores in 28 joints (DAS28) were lower in patients with RA and severe ILD than in those without ILD at RA presentation [19]. Some of this data conflicted with our study's finding that RA patients with ILD had significantly higher baseline CRP levels. This discrepancy might be due to the fact that in the present study, we did not include whole components of DAS28 such as swollen joint count, tender joint count, and patient global assessment as a measure of arthritis activity.

RA patients with ILD had received MTX less frequently and sulfasalazine more frequently than those without ILD. These results may be due to the potential lung toxicity of MTX. In addition, in subgroup analysis of RA patients with ILD, any medications, including MTX, were not significantly different between living and deceased groups. These results suggest that MTX may not significantly affect the long-term survival of patients with ILD in RA.

A review paper by Assayag et al. [20] found that the significant predictors of mortality in RA patients with ILD were older age, male gender, lower diffusing capacity of the lung for carbon dioxide (DLCO), increased extent of fibrosis, and presence of the UIP subtype. In this study, older age at the time of the diagnosis of ILD was significantly associated with mortality in RA patients with ILD. This finding was consistent with the results of previous studies that suggested that lower performance status and older age were predictors of mortality in RA patients with ILD group [21,22]. The results of this study suggest that the UIP subtype on HRCT did not show a statistically significant association but could be associated with a poor prognosis.

No correlation of survival with smoking status or baseline lung function was found in this RA with ILD patient population. However, a previous study reported that those factors are predictors of the progression of ILD in systemic sclerosis-associated ILD [23,24]. Unfortunately, we could not analyze further due to lack of regular follow-up data of lung function.

The present study had some limitations. First, data on the number of swollen and tender joints and patient global assessment, which is representative of RA disease activity, could not be analyzed due to the retrospective study design. However, ESR and CRP levels were assessed, and these variables are known to be associated with disease activity over time. Second, the patients in this study did not have regular pulmonary function test follow-up. Third, this study utilized a relatively small number of patients recruited from a single center.

In conclusion, RA patients with ILD had higher RF and anti-CCP titers and baseline CRP levels than those without ILD. The survival of RA patients with ILD is adversely affected by age at the time of ILD diagnosis. RA-ILD patients diagnosed after age 65 or with a UIP subtype on HRCT may have a poor prognosis. A larger prospective study is needed to confirm the present findings.

\section{KEY MESSAGE}

1. Rheumatoid arthritis (RA) patients with interstitial lung disease (ILD) had higher rheumatoid factor and anti-cyclic citrullinated peptide titers and baseline C-reactive protein levels than those without ILD.

2. Older age at the time of the ILD diagnosis was significantly associated with mortality of RA patients with ILD.

3. Usual interstitial pneumonia subtype on high-resolution computed tomography may be a poor prognostic factor among RA patients with ILD. 


\section{Conflict of interest}

This work was supported by JW Pharmaceutical (JWP) ISP2013-001.

\section{REFERENCES}

1. Kim EJ, Collard HR, King TE Jr. Rheumatoid arthritis-associated interstitial lung disease: the relevance of histopathologic and radiographic pattern. Chest 2009;136:1397-1405.

2. O'Dwyer DN, Armstrong ME, Cooke G, Dodd JD, Veale DJ, Donnelly SC. Rheumatoid arthritis (RA) associated interstitial lung disease (ILD). Eur J Intern Med 2013;24:597-603.

3. Mori S, Koga Y, Sugimoto M. Different risk factors between interstitial lung disease and airway disease in rheumatoid arthritis. Respir Med 2012;106:1591-1599.

4. Park JH, Kim DS, Park IN, et al. Prognosis of fibrotic interstitial pneumonia: idiopathic versus collagen vascular disease-related subtypes. Am J Respir Crit Care Med 2007;175:705-711.

5. Kim EJ, Elicker BM, Maldonado F, et al. Usual interstitial pneumonia in rheumatoid arthritis-associated interstitial lung disease. Eur Respir J 2010;35:1322-1328.

6. Fischer A, du Bois R. Interstitial lung disease in connective tissue disorders. Lancet 2012;380:689-698.

7. Schoe A, van der Laan-Baalbergen NE, Huizinga TW, Breedveld FC, van Laar JM. Pulmonary fibrosis in a patient with rheumatoid arthritis treated with adalimumab. Arthritis Rheum 2006;55:157-159.

8. Perez-Alvarez R, Perez-de-Lis M, Diaz-Lagares C, et al. Interstitial lung disease induced or exacerbated by TNF-targeted therapies: analysis of 122 cases. Semin Arthritis Rheum 2011;41:256-264.

9. Arnett FC, Edworthy SM, Bloch DA, et al. The American Rheumatism Association 1987 revised criteria for the classification of rheumatoid arthritis. Arthritis Rheum 1988;31:315-324.

10. Travis WD, Costabel U, Hansell DM, et al. An official American Thoracic Society/European Respiratory Society statement: update of the international multidisciplinary classification of the idiopathic interstitial pneumonias. Am J Respir Crit Care Med 2013;188:733-748.

11. Giles JT, Danoff SK, Sokolove J, et al. Association of fine specificity and repertoire expansion of anticitrullinated peptide antibodies with rheumatoid arthritis associated interstitial lung disease. Ann Rheum Dis 2014;73:1487-1494.
12. Zhu J, Zhou Y, Chen X, Li J. A metaanalysis of the increased risk of rheumatoid arthritis-related pulmonary disease as a result of serum anticitrullinated protein antibody positivity. J Rheumatol 2014;41:1282-1289.

13. Shachor Y, Schindler D, Siegal A, Lieberman D, Mikulski Y, Bruderman I. Increased incidence of pulmonary tuberculosis in chronic interstitial lung disease. Thorax 1989;44:151-153.

14. Silva I, Mateus M, Branco JC. Assessment of erythrocyte sedimentation rate (ESR) and C- reactive protein (CRP) on rheumatoid arthritis activity prediction. Acta Reumatol Port 2010;35:456-462.

15. Koduri G, Norton S, Young A, et al. Interstitial lung disease has a poor prognosis in rheumatoid arthritis: results from an inception cohort. Rheumatology (Oxford) 2010;49:1483-1489.

16. Liu X, Mayes MD, Pedroza C, et al. Does C-reactive protein predict the long-term progression of interstitial lung disease and survival in patients with early systemic sclerosis? Arthritis Care Res (Hoboken) 2013;65:1375-1380.

17. Mortensen RF. C-reactive protein, inflammation, and innate immunity. Immunol Res 2001;24:163-176.

18. Ohtsuka T. Serum interleukin-6 level is reflected in elevated high-sensitivity C-reactive protein level in patients with systemic sclerosis. J Dermatol 2010;37:801-806.

19. Chan E, Chapman K, Kelly C. Interstitial lung disease in rheumatoid arthritis: a review. Arthritis Res UK 2013;3:1-9.

20. Assayag D, Lubin M, Lee JS, King TE, Collard HR, Ryerson CJ. Predictors of mortality in rheumatoid arthritis-related interstitial lung disease. Respirology 2014;19:493-500.

21. Migita K, Arai T, Jiuchi Y, et al. Predictors of mortality in patients with interstitial lung disease treated with corticosteroids: results from a cohort study. Medicine (Baltimore) 2014;93:e175.

22. Olson AL, Swigris JJ, Sprunger DB, et al. Rheumatoid arthritis-interstitial lung disease-associated mortality. Am J Respir Crit Care Med 2011;183:372-378.

23. Saag KG, Kolluri S, Koehnke RK, et al. Rheumatoid arthritis lung disease: determinants of radiographic and physiologic abnormalities. Arthritis Rheum 1996;39:1711-1719.

24. Hudson M, Steele R; Canadian Scleroderma Research Group, Baron M. Immunosuppression for interstitial lung disease in systemic sclerosis: novel insights and opportunities for translational research. J Cell Commun Signal 2012;6:187-190. 